\title{
Caracterización y clasificación de la bahía de Puerto Montt mediante batimetría de multihaz y datos de backscatter
}

\author{
Cristián Rodrigo ${ }^{1}$ \\ ${ }^{1}$ Servicio Hidrográfico y Oceanográfico de la Armada de Chile \\ Casilla 324, Valparaíso
}

\begin{abstract}
RESUMEN. Se efectuó un levantamiento batimétrico de alta resolución con sonar multihaz en la bahía de Puerto Montt, Chile. Además se obtuvo, con el mismo sistema de sonar, datos de intensidad de las señales acústicas reflejadas sobre el fondo del mar y se calculó la razón entre la energía acústica emitida y la recibida en los transductores (backscatter o retrodispersión acústica). El modelo topográfico resultante presentó una suave plataforma costera y una vertiente submarina abrupta. Existen pequeños canales submarinos en la zona de la vertiente y en la zona central de la bahía hay un canal principal que puede ser relacionado con la depresión que da origen al canal Tenglo. Estos canales han servido de conducto para el transporte de sedimentos desde el sector somero al profundo. La información de backscatter se utilizó para clasificar los sedimentos del fondo apoyado con los resultados de muestras de sedimentos. Se encontró que los sedimentos más gruesos tienen mayor intensidad o fuerza de backscatter que los finos. En la bahía predominan las arenas fangosas, pero los materiales más gruesos tienden a concentrarse en sectores someros y los más finos en sectores profundos. En el interior y en las bocas del canal Tenglo, el transporte de sedimentos es fuertemente influenciado por las corrientes de marea, no así el transporte de materiales sobre las vertientes submarinas y el área más profunda de la bahía, donde otros mecanismos, como corrientes de turbidez, tendrían más importancia.
\end{abstract}

Palabras clave: batimetría multihaz, retrodispersión acústica, sedimentos marinos, topografía submarina, Puerto Montt, Chile.

\section{Seabed characterization and classification in Puerto Montt bay using multibeam bathymetry and backscatter data}

\begin{abstract}
A high resolution multibeam bathymetric survey was carried out in Puerto Montt bay, Chile. Moreover, intensity data derived from acoustic signals reflected by the sea bottom was gathered using the same sonar system; the ratio was calculated between emitted and backscattered energy. The resulting topographic model shows a soft coastal platform and an abrupt slope. There are small submarine channels in the slope zone and a main channel in the central bay zone that could be related to the depression associated with the Tenglo channel. These channels act as conduits for the sediment transport from shallow to deep waters. The backscatter information was used in order to classify seabed sediments, subsequently confirmed by analyzing sediment samples from the respective sites. Coarse sediments feature higher intensities or backscatter signals than fine sediments. Muddy sands generally prevail in the investigation area. However, coarse material tends to concentrate in shallow waters and fine material in deep sectors. Sediment transport in the interior and the mouths of Tenglo channel in strongly influenced by tide currents. This does not hold for material transport occurring on the slope and in the deeper parts of the bay, where other mechanisms, such as turbidity currents, are more important.
\end{abstract}

Key words: multibeam bathymetry, backscatter data, marine sediments, submarine topography, Puerto Montt, Chile.

Autor corresponsal: Cristián Rodrigo (crodrigo@inach.cl).

Dirección actual: Departamento Científico, Instituto Antártico Chileno, Plaza Muñoz Gamero 1055, Punta Arenas.

\section{INTRODUCCIÓN}

Hace dos décadas que se utilizan los sistemas de sonar multihaz para determinar la topografía submarina en forma detallada. A diferencia de un ecosonda tra- dicional (un haz), un sonar multihaz crea varios haces de sonido que se disponen en forma de abanico bajo los transductores, los cuales barren el fondo del mar 
transversalmente a la trayectoria de navegación, obteniéndose datos de profundidad con alta resolución. Esto permite efectuar levantamientos batimétricos sobre grandes áreas a un costo relativamente bajo. En un comienzo estos sistemas se aplicaban sólo para estudios científicos relacionados con la exploración geofísica de aguas profundas pero, por el desarrollo tecnológico se mejoraron los sistemas computacionales, algoritmos de proceso de señales, sensores de movimiento y otros componentes, que permiten sobrepasar las exigencias de calidad internacionales para el levantamiento batimétrico. Como consecuencia, se produjo un aumento de los levantamientos con sonares multihaz en aguas costeras, para satisfacer objetivos hidrográficos e ingenieriles.

Los sistemas de sonar multihaz entregan datos de profundidades georreferenciadas, y datos de las amplitudes o intensidad de las señales acústicas reflejadas en el fondo del mar hacia los transductores (retrodispersión acústica, más conocida como señal de backscatter). Esta información se registra en un sonograma (imagen acústica, en modo sonar de rebusca lateral o side scan sonar) y es muy utilizada para la detección de objetos sobre el fondo marino (rocas, naufragios, tuberías, etc.). Si además estos datos son apropiadamente reducidos, proveen una medida de la fuerza del backscatter como una función del ángulo de elevación (del rayo o haz acústico) o respuesta angular (Hughes-Clarke et al., 1997). En este trabajo se utiliza el concepto de backscatter como la razón entre la energía acústica emitida y la recibida en los transductores, según se define en el Atlas Electronics (2001). Las variaciones de la fuerza del backscatter son producidas principalmente por los cambios de las impedancias acústicas asociadas a cambios en los tipos superficiales de fondo (arena, roca, fango, etc.).

Los tipos de fondo marino se determinan por análisis granulométrico y la distribución espacial se define por interpolación o extrapolación entre muestras. Sin embargo, la obtención de la distribución detallada de los tipos de fondo difícilmente se alcanza, porque generalmente se recolecta baja cantidad de muestras para describir adecuadamente la variabilidad de los tipos superficiales de fondo (Dartnell \& Gardner, 2004). Por lo tanto, con los datos de backscatter proporcionados por un sistema de sonar multihaz y calibrados por el conocimiento del tipo de fondo en puntos específicos, se puede estimar en detalle la calidad o tipo de fondo marino en un área dada y construir un mapa de su distribución espacial.
Este tipo de información puede ser crucial cuando se modelan procesos sedimentarios, transporte de contaminantes y en la definición de hábitat bentónicos (Dartnell \& Gardner, 2004).

En este trabajo se establecen las características principales de la topografía submarina y de la distribución superficial de sedimentos en la bahía de Puerto Montt, a través del análisis de datos batimétricos y de la información de backscatter de un sonar multihaz. Este es el primer estudio de este tipo efectuado en la bahía de Puerto Montt y el primero que utiliza información de backscatter, en un análisis sedimentológico en un área costera de Chile.

\section{MATERIALES Y MÉTODOS}

En enero de 2002 se realizó un levantamiento batimétrico de alta resolución con sonar multihaz en la bahía de Puerto Montt (Fig. 1), para obtener datos hidrográficos actualizados y de mejor calidad para la confección de la carta náutica de la bahía. En forma paralela al sondaje batimétrico, se obtuvo con el mismo sistema, datos de intensidad de las señales acústicas reflejadas sobre el fondo del mar (modo side scan sonar).

El sondaje se realizó con el sistema de sonar multihaz Atlas Hydrosweep MD-2, instalado en el buque PSH "Cabrales" de la Armada de Chile. Este sistema genera 320 haces de sonido con pulsos de $50 \mathrm{kHz}$ de frecuencia, con una cobertura lateral de hasta seis veces la profundidad (sondaje estándar). El posicionamiento fue de tipo satelital diferencial (DGPS). Antes de comenzar con el sondaje, se efectuó el lance de una sonda perfiladora del sonido en un área representativa. Esta sonda mide directamente la velocidad del sonido en profundidad. De este modo se calibró el sonar multihaz según la estructura de velocidad del sonido de la columna de agua.

Durante el levantamiento, la posición del área del piso marino sonificada por cada haz se corrigió con la información proveniente del sensor de movimiento que mide los ángulos de actitud del buque (cabeceo, balanceo, guiñada y altura sobre o bajo el nivel medio del mar). Además, las señales acústicas se corrigieron constantemente por las variaciones de la velocidad del sonido superficial. En la Figura 2 se indican las trayectorias de navegación (tracks) efectuadas en el área de estudio.

Como complemento para algunas de las figuras de los resultados, se insertó una imagen del relieve 


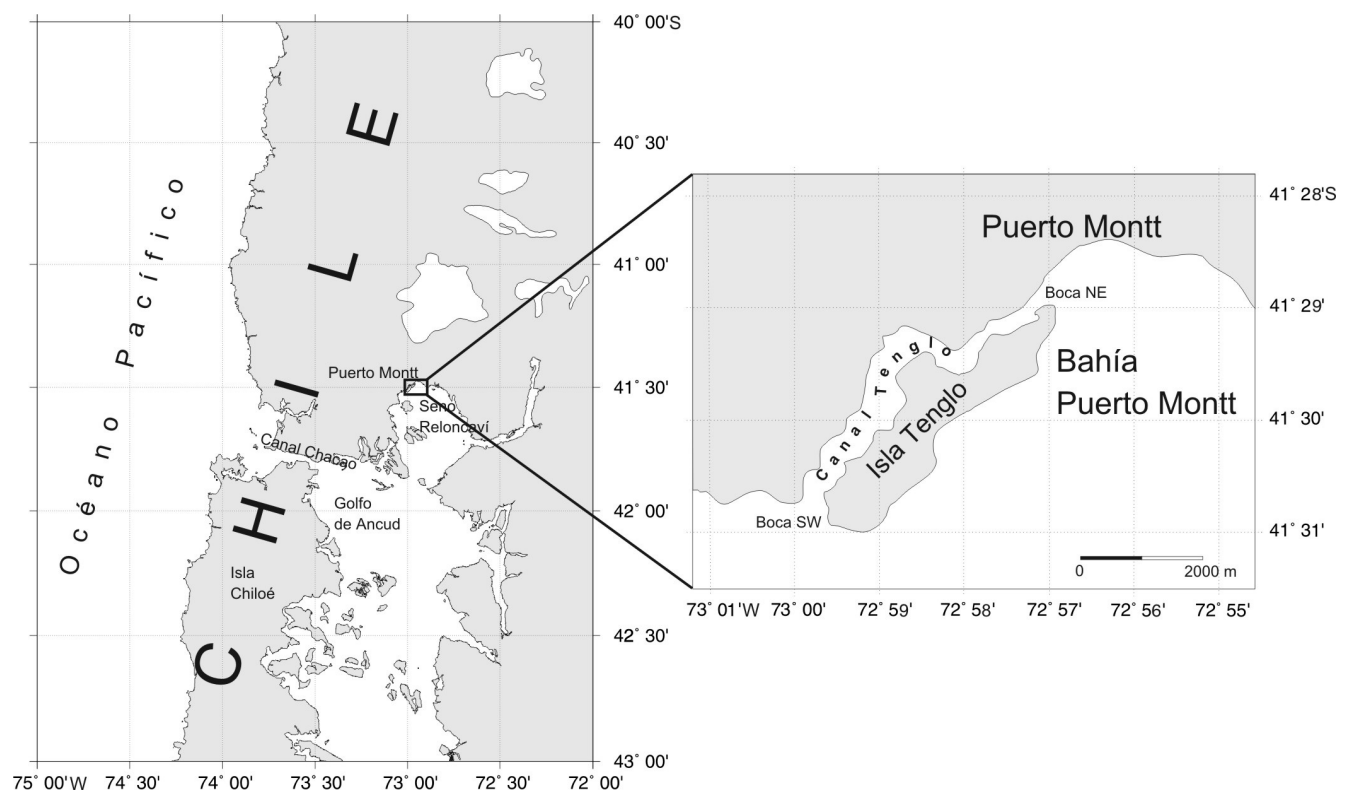

Figura 1. Ubicación geográfica de la bahía de Puerto Montt.

\section{Figure 1. Geographic location of Puerto Montt bay.}

terrestre e iluminada (desde el norte y con elevación de $50^{\circ}$ ), confeccionada a partir de datos de topografía derivados de altimetría de radar del trasbordador espacial Endeavour, obtenidos en el año 2000, del proyecto "Shuttle Radar Topography Mission (SRTM)" de la NASA (Rabus et al., 2003). Esta topografía terrestre tiene una resolución de 3' de arco.

El postproceso de la batimetría consistió en la corrección y verificación de los datos de posición, movimientos del buque y velocidad del sonido; en la eliminación de puntas batimétricas anómalas (spikes), corrección de artificios batimétricos y filtro de ruido. Los datos batimétricos fueron corregidos por marea, cuyos datos provinieron de la estación permanente del Servicio Hidrográfico y Oceanográfico de la Armada de Chile (SHOA) en Puerto Montt.

Se elaboró un modelo de elevación digital con una resolución de $8 \times 8 \mathrm{~m}$ con los datos batimétricos postprocesados. Los datos del modo side scan sonar no fueron postprocesados, ya que se consideraron sólo como apoyo a la interpretación y se creó un mosaico de los sonogramas crudos con una resolución de $5 \times 5 \mathrm{~m}$. Se buscó el mejor contraste de tonos de grises en las imágenes de los sonogramas digitales, para su mejor representación. La fuerza del backscatter se obtuvo procesando los datos con el programa de Atlas Electronics (2001), que calcula la razón de energía entre el haz emitido y el recibido, corregida por la distancia entre los transductores y el piso marino. La fuerza del backscatter se grafica en decibeles versus la respuesta angular o ángulo de crecimiento (Fig. 3).

La batimetría se puede interpretar usando principios de observación topográfica directos, sin embargo, la interpretación del backscatter es más complicada porque representa una interacción compleja entre el pulso acústico en el piso del mar, así como en el subfondo. La fuerza del backscatter depende del nivel acústico de la fuente, frecuencia usada (en este caso $50 \mathrm{kHz}$ ), ángulo de crecimiento, densidad de los materiales, rugosidad del piso marino y reverberaciones de volumen dentro de unos pocos metros (Urick, 1983; Gardner et al., 1991; Augustin et al., 1996; Dartnell \& Gardner, 2004).

Para realizar una asociación más acertada entre la fuerza del backscatter y los tipos superficiales de fondo, se tomaron 42 muestras de sedimentos con draga en la bahía (Fig. 4). Estas muestras se tamizaron según el método estándar y se clasificaron granulométricamente de acuerdo a la escala de Wentworth (1922). Posteriormente, se reclasificaron de acuerdo a la terminología textural de Folk et al. (1970). El tipo de sedimento de cada estación se asoció a la fuerza del backscatter correspondiente a esa posición, calculada por el sistema. Este valor correspondió al promedio de los valores de varias emisiones/recepciones del sistema (alrededor de 10), en el sector de la estación o punto de muestreo, para 


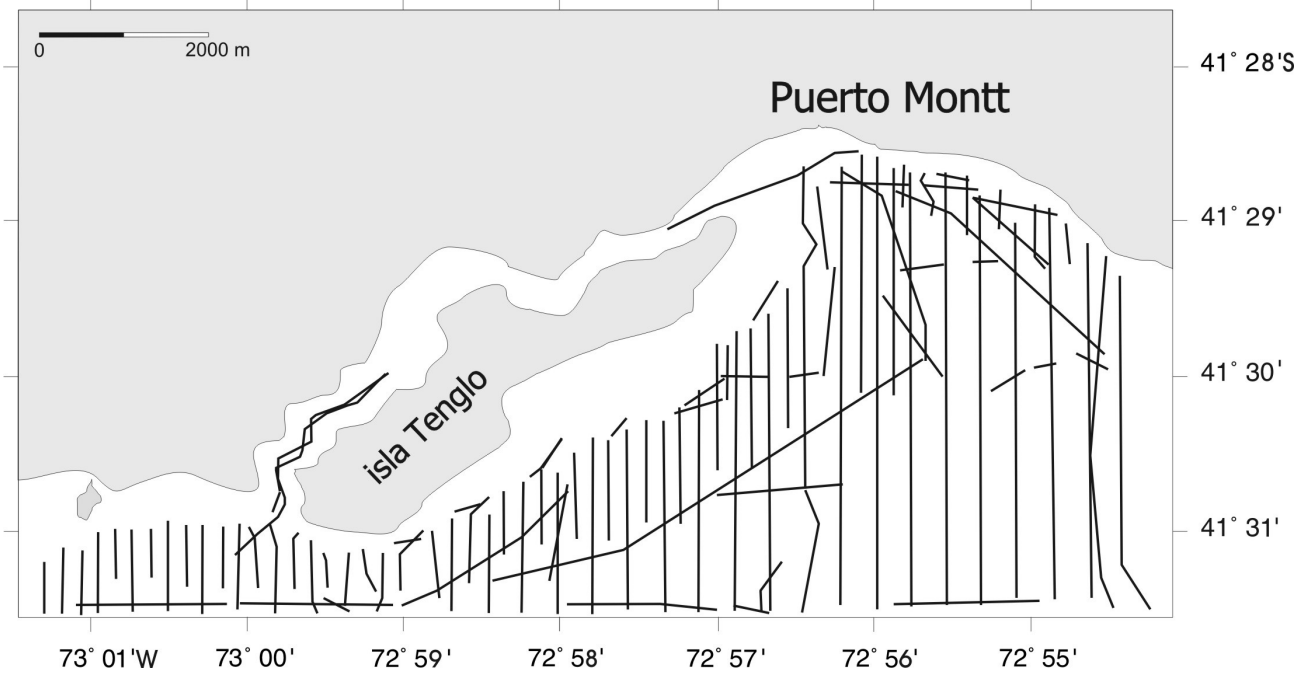

Figura 2. Trayectorias de navegación efectuadas en el levantamiento batimétrico de la bahía de Puerto Montt.

Figure 2. Navigation tracks done during bathymetric survey in Puerto Montt Bay.
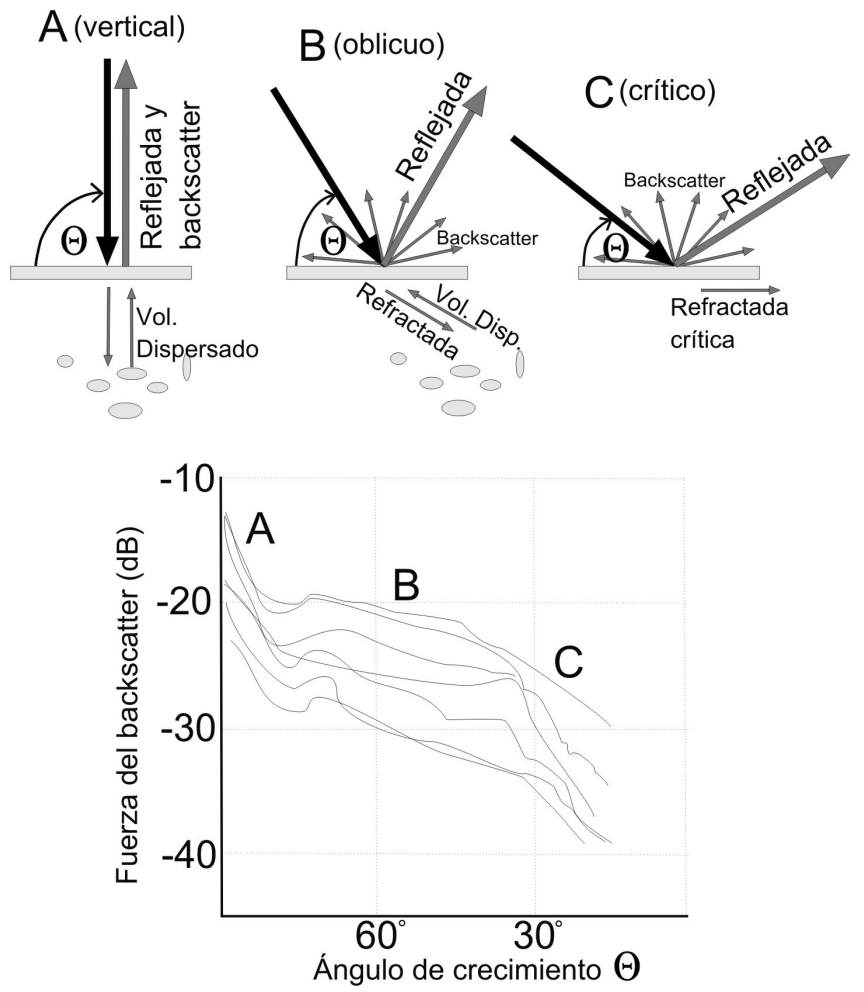

Figura 3. Relación entre la fuerza del backscatter y el ángulo de crecimiento del haz acústico emitido. En un sistema de sonar multihaz, el sector de haces centrales estaría representado por " $A$ " y los laterales por " $C$ ". Las distintas curvas que aparecen en el gráfico, indican que la fuerza del backscatter es variable (modificado de De Moustier, 2002).

Figure 3. Relationship between backscatter strength and acoustic beam grazing angle emitted. In a multibeam sonar system, central beams are represented by " $\mathrm{A}$ " and lateral beams by " $\mathrm{C}$ ". The different graphic curves, show that backscatter strength is variable (modified after De Moustier, 2002). 
eliminar parcialmente el posible ruido. La Figura 5 muestra un gráfico con ejemplos de las asociaciones entre curvas de la fuerza del backscatter y los diferentes tipos de sedimentos (De Moustier, 2002). A continuación se preparó una lista de las asociaciones para el área y se realizó una clasificación automática (supervisada) para todo el cubrimiento acústico obtenido con el sistema multihaz, resultando un mapa continuo de la distribución de los tipos superficiales de fondo.

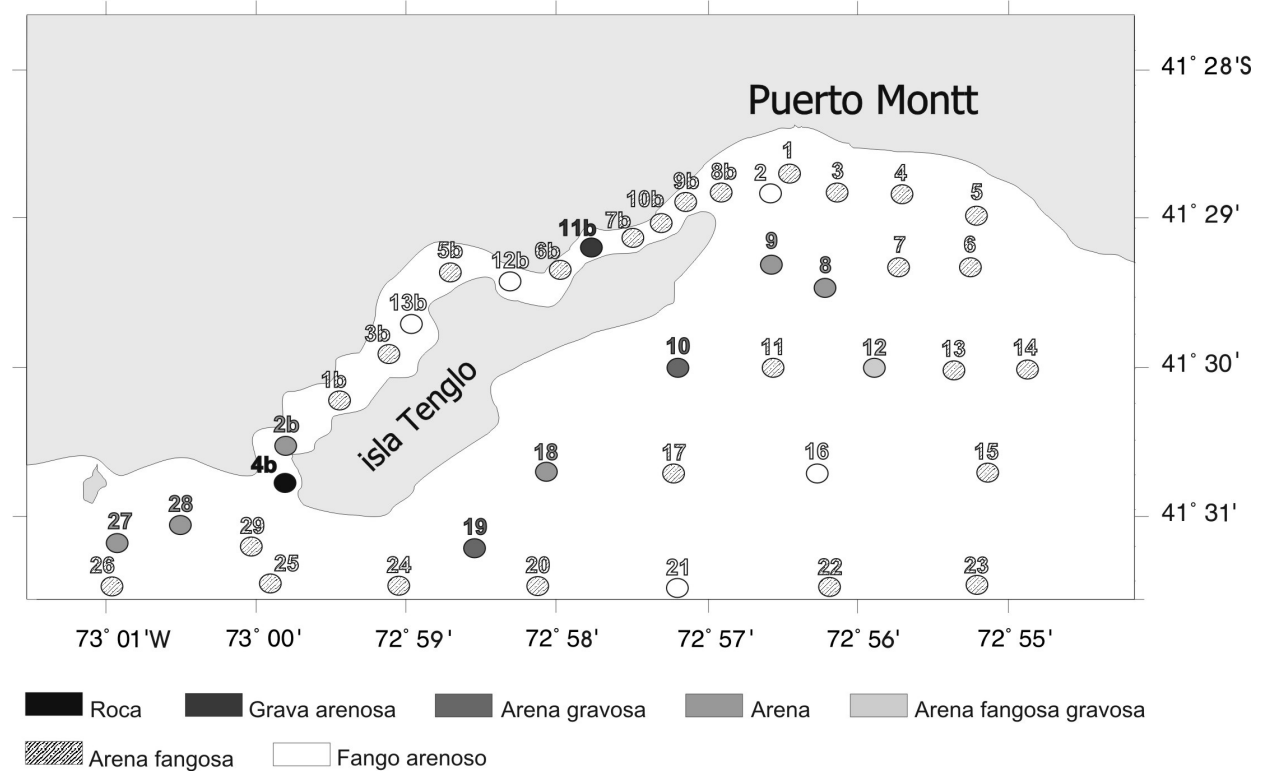

Figura 4. Estaciones de muestreo de sedimentos y tipificación según la clasificación textural de Folk et al. (1970).

Figure 4. Sediment sample stations and classification using textural classification of Folk et al. (1970).

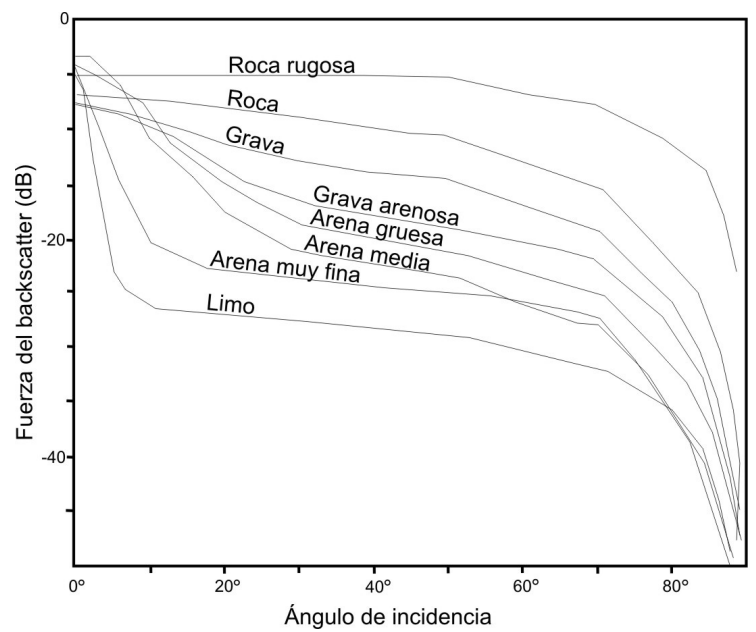

Figura 5. Relación entre intensidad de backscatter y ángulo de incidencia, según tamaño de grano de sedimento, para una frecuencia de $30 \mathrm{kHz}$ (modificado de De Moustier, 2002).

Figure 5. Relationship between backscatter strength and incidence angle, according sediment grain size, for a 30 kHz frequency (modified after De Moustier, 2002). 


\section{RESULTADOS}

\section{Geomorfología Submarina}

El fondo marino de la bahía de Puerto Montt se compone de una zona profunda y otra somera. La somera corresponde a una plataforma costera en el sector oriental de la isla Tenglo, que es de pendiente suave hasta una profundidad cercana a $50 \mathrm{~m}$ (Fig. 6). La costa oriental de la bahía no presenta una plataforma extensa en relación a la occidental de isla Tenglo y cae con una vertiente submarina pronunciada hasta aproximadamente $200 \mathrm{~m}$. La vertiente submarina que rodea la isla, también cae con una pendiente abrupta hacia la bahía hasta $200 \mathrm{~m}$. A continuación de la vertiente submarina, existe la zona profunda que se ubica al centro de la bahía, que tiene una pendiente moderada en comparación con la de la vertiente y alcanza una profundidad máxima de unos $330 \mathrm{~m}$.

La plataforma presenta varios rasgos abultados, en el sector NE de la isla Tenglo y en la boca SW del canal Tenglo. A menor escala, el sistema multihaz permitió detectar ondas de fondo al interior del canal (Fig. 7), cuyas crestas se orientan en forma perpendicular con respecto a las riberas del canal.
La vertiente submarina se presenta rugosa, marcada por varias depresiones angostas (Fig. 6) y en el borde superior de la vertiente con la plataforma, alrededor de la isla, a una profundidad aproximada de $100 \mathrm{~m}$, se observan varios montículos orientados con tendencia NW-SE. En la vertiente de la salida SW del canal Tenglo, se detecta otro sector abultado del piso marino, creándose dos pequeñas terrazas o escalonamientos.

El área profunda tiene en el centro una depresión angosta que recorre toda la bahía. En la parte norte tiene dirección W-E y nace en la boca NE del canal Tenglo. Después cambia su rumbo bruscamente de W-E a N-S cerca de la longitud $72^{\circ} 55^{\prime} 12^{\prime}$ 'W. Más al sur esta depresión cambia nuevamente de rumbo y se curva suavemente hacia el SE. En esa misma área, a una profundidad de $240 \mathrm{~m}$, existe otro abultamiento del piso marino, que tiene una forma elíptica convexa, cuyo eje mayor tiene rumbo NW-SE. En general, el área profunda carece de rasgos, excepto los mencionados y presenta algunas prolongaciones de depresiones menores provenientes de la vertiente occidental.

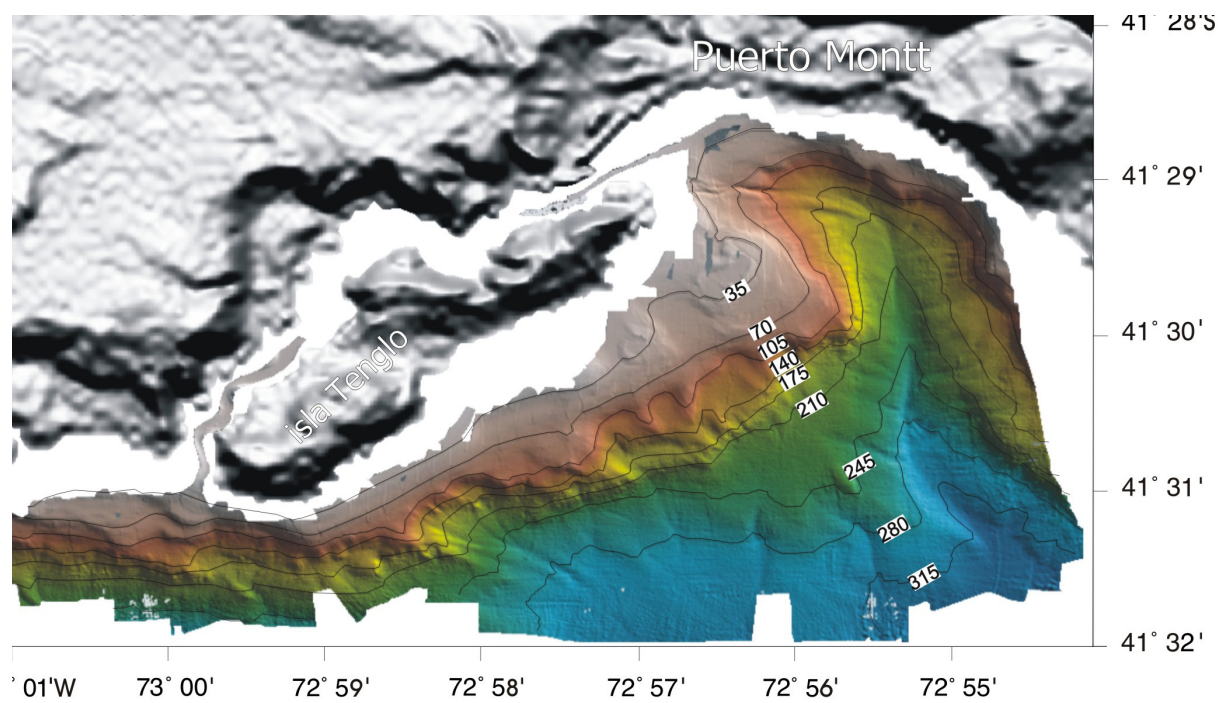

Figura 6. Mapa de la topografía submarina a partir de datos de sonar multihaz. Contornos batimétricos cada $35 \mathrm{~m}$.

Figure 6. Submarine topography map from multibeam sonar data. Bathymetric contours every 35 m. 


\section{Mosaico de side scan sonar}

El mosaico del modo side scan sonar obtenido al unir los sonogramas crudos de la intensidad de las señales acústicas del sistema multihaz, muestra que las áreas obscuras tienen mayor intensidad y las claras menor (Fig. 8). En esta escala sólo se pueden apreciar los rasgos mayores del fondo marino como la depresión central en la zona profunda, el borde del pie de la vertiente submarina y el contacto de la plataforma con la zona rugosa de la vertiente submarina, identificadas por las altas intensidades acústicas. En general, predominan las intensidades intermedias y, exceptuando las áreas mencionadas, son relativamente uniformes. En este estudio, no se tiene como objetivo el análisis detallado de la información de side scan. Sin embargo, cabe destacar que es posible detectar objetos mayores como barcos o pequeños como tuberías metálicas o depresiones sobre el fondo producto del movimiento de anclas (Fig. 7).

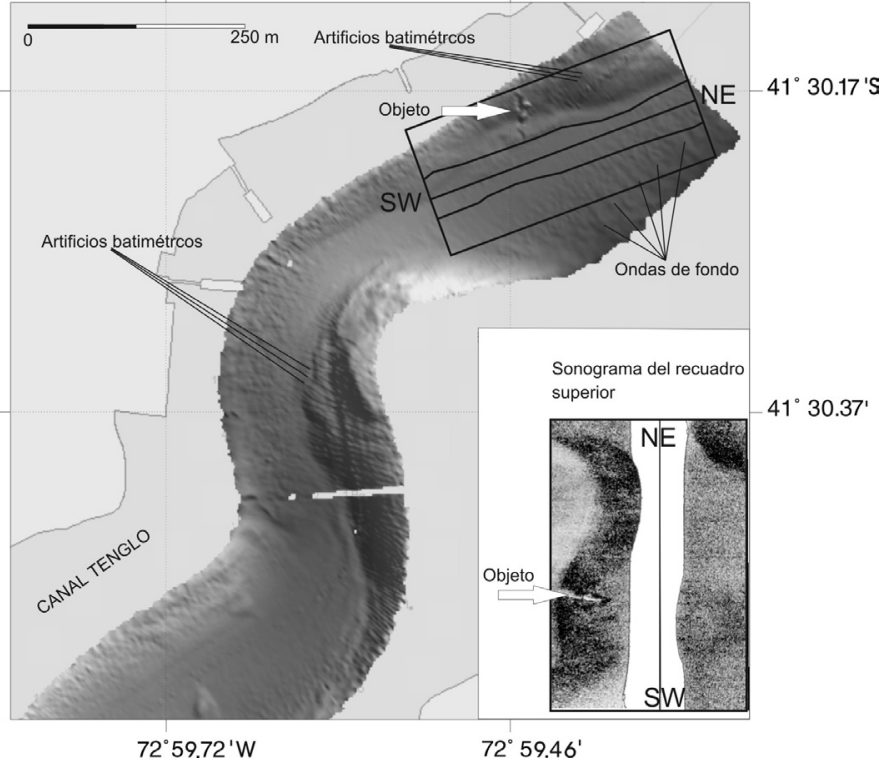

Figura 7. Carta morfológica (resolución $2 \times 2 \mathrm{~m}$ ) en un sector del canal Tenglo, confeccionada mediante datos de batimetría multihaz. La flecha blanca indica la detección de un naufragio o restos metálicos. Estos restos son detectados en el sonograma correspondiente (al costado inferior derecho).

Figure 7. Morphological chart $(2 \times 2$ $m$ resolution) in a Tenglo channel sector, done from multibeam bathymetry data. White arrow indicate shipwreck or metallic rests. These rests are detected in the correspondent sonogram (to the lower right side).

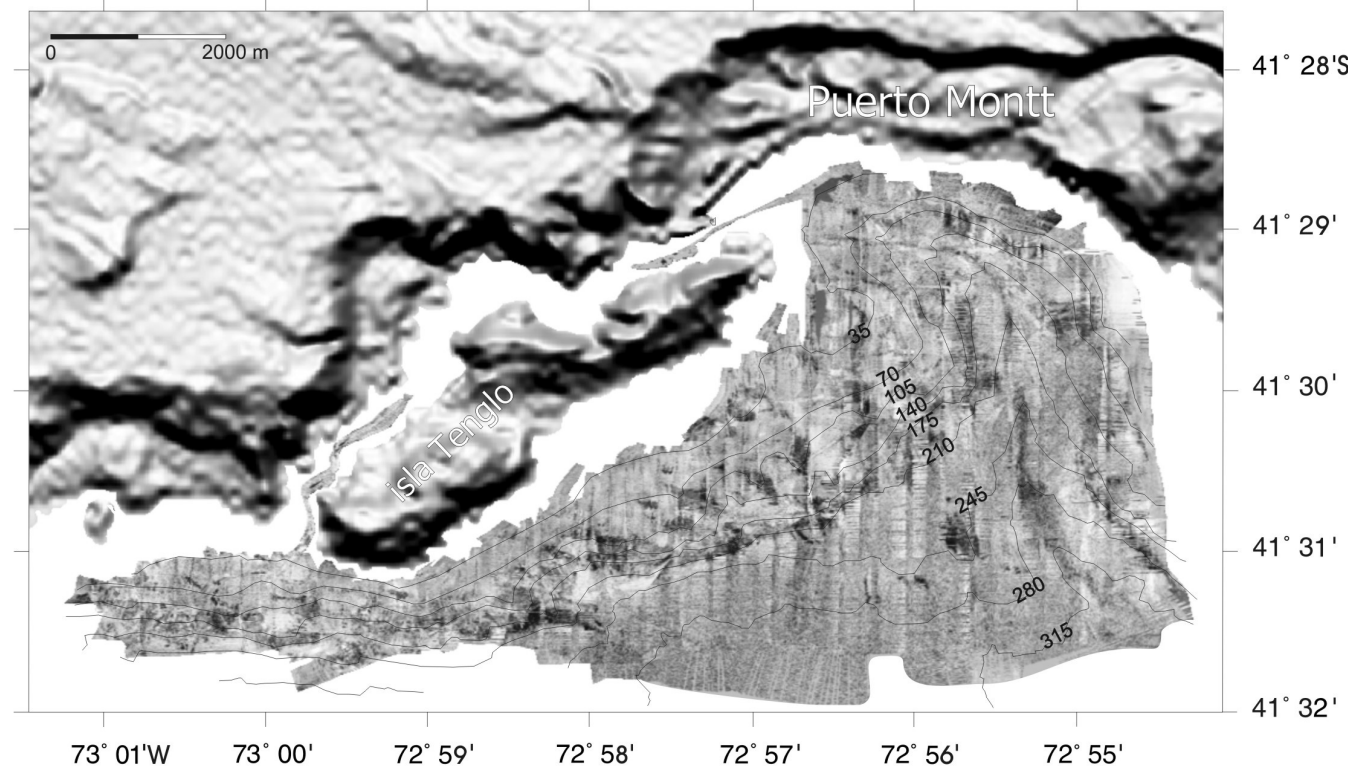

Figura 8. Mosaico de los sonogramas crudos (modo side scan sonar) obtenidos con el sonar multihaz.

Figure 8. Crude sonogram mosaic (side scan sonar mode) obtained by the multibeam system. 


\section{Clasificación de los tipos de sedimentos superficiales del fondo marino}

La granulometría del sedimento muestra un predominio de arenas, como puede comprobarse de los resultados de 12 estaciones consideradas como representativas (Fig. 9). En el canal Tenglo se encuentra que los granos más gruesos se distribuyen en los extremos del canal y los más finos al interior (Fig. 4). Las estaciones $3 \mathrm{~b}$ y $6 \mathrm{~b}$ se encuentran a distancia similar a partir del centro del canal hacia las bocas; sin embargo, en la estación $3 b$ predominan los limos-arcillas y en la $6 \mathrm{~b}$ la arena fina, casi en proporciones iguales (Fig. 9). En la bahía de Puerto
Montt, los patrones de distribución de sedimentos son los habituales, los materiales más gruesos están más cerca de la costa y los más finos, en los sectores de mayor profundidad. Las estaciones 4, 8, 18 y 27 , se localizan sobre el sector de plataforma y las muestras poseen similar distribución de clases, encontrándose un predominio de arena fina y mediana. A diferencia de aquellas ubicadas sobre la vertiente submarina (ej. estación 19), donde se encuentran materiales finos y gruesos mezclados. En la zona profunda (ej. estaciones 20, 21 y 24), predominan los limos-arcillas, mezclados con arenas, en especial, en los sectores más próximos a la vertiente submarina.

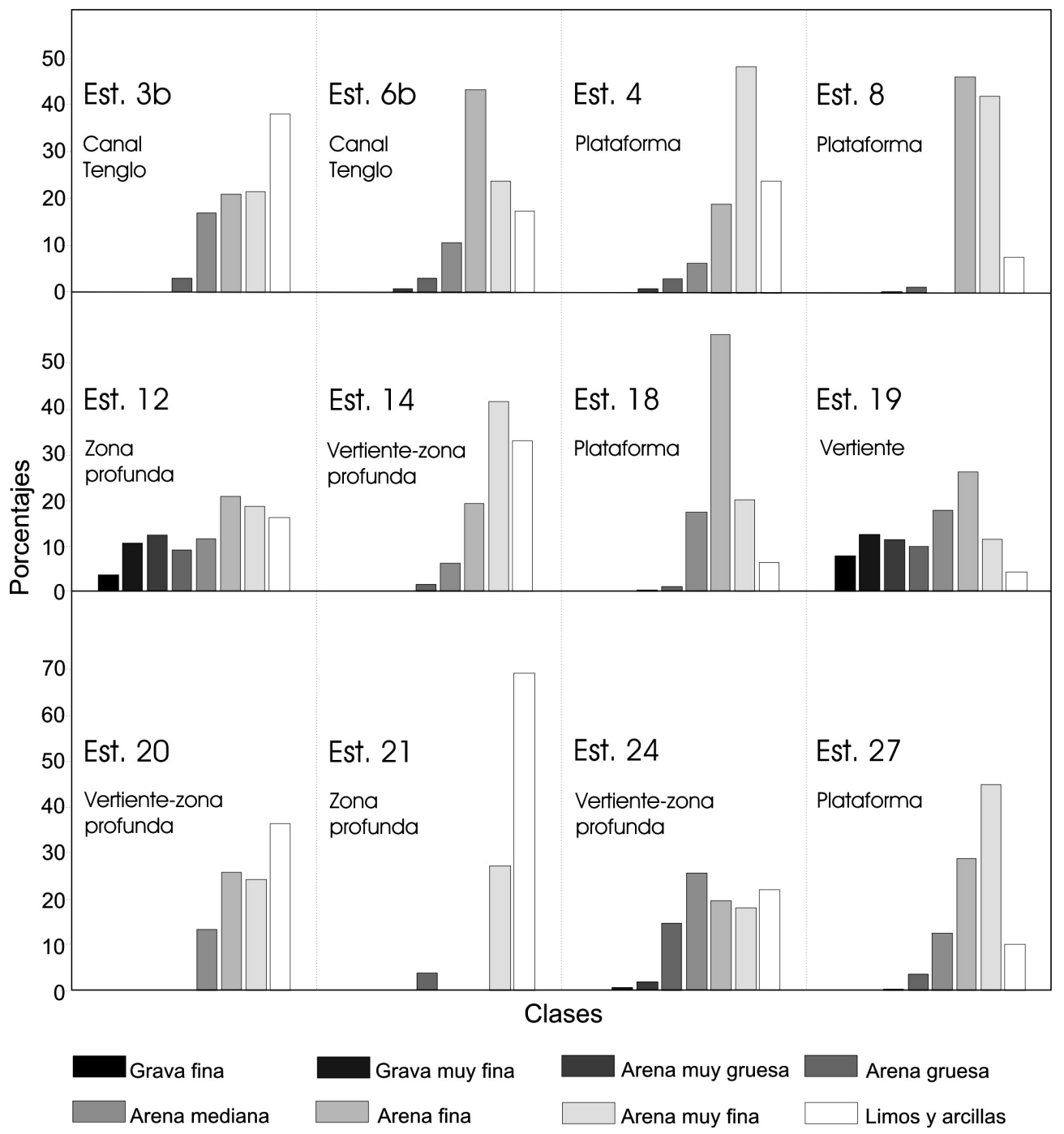

Figura 9. Distribución granulométrica de acuerdo a la escala de Wentworth (1922), para muestras de sedimentos en algunas estaciones representativas.

Figure 9. Grain size distribution according Wentworth (1922) scale, for sediment samples of some representative stations. 
Se observa que en la bahía los sedimentos tipo arena o más gruesos se encuentran principalmente sobre la plataforma y los más finos en la zona profunda (Fig. 10). En esta zona predomina la arena fangosa, pero existen áreas de materiales más finos de fangos arenosos, localizadas principalmente a continuación de sectores con depresiones angostas en la vertiente submarina.

La clasificación textural de las muestras no mostró fango. Sin embargo, el sistema indicó niveles de backscatter menores al fango arenoso, que fueron clasificados como fango. Se encontraron tres áreas con este tipo de sedimentos, una en el centro de la bahía $\left(41,50^{\circ} \mathrm{S}, 72,91^{\circ} \mathrm{W}\right)$, otra al SW de la boca SW del canal Tenglo y un área pequeña al E de la boca del canal (Fig. 10).

\section{DISCUSIÓN}

El fondo marino de la bahía de Puerto Montt se compone de una serie de rasgos topográficos a diversas escalas. Los rasgos principales a mayor escala son una plataforma costera que tiene su parte más ancha al costado oriental de la isla Tenglo, una vertiente submarina caracterizada por numerosos canales submarinos y una zona profunda, de pendiente moderada, que en el centro posee un canal submarino principal.

A pequeña escala se encontraron ondas de fondo en el sector del canal Tenglo (Fig.7), que indican la presencia de corrientes marinas que modifican la forma del piso marino. Estas ondas sugieren transporte de sedimentos en el interior del canal, donde las corrientes transportan materiales hacia dentro o fuera, influyendo sobre el patrón sedimentario de la bahía. En la parte norte del canal, las corrientes son hacia el oeste en flujo y al este en reflujo (SHOA, 2001), sugiriendo que las corrientes están directamente asociadas a la marea.

La existencia de distintos tipos de sedimentos al interior del canal sugieren patrones desiguales de depositación, ya sea por una interacción compleja de los granos sedimentarios ante las características de las corrientes de marea, fenómenos de erosión y transporte por corrientes o por diferentes tipos de descarga de materiales sobre el canal, como el aporte por escurrimiento superficial causado por lluvias o desagües domésticos o industriales.

A una distancia aproximada de $1000 \mathrm{~m}$ hacia fuera de ambas bocas del canal, existe una zona de material más fino (fango en Fig. 10). Estos fangos

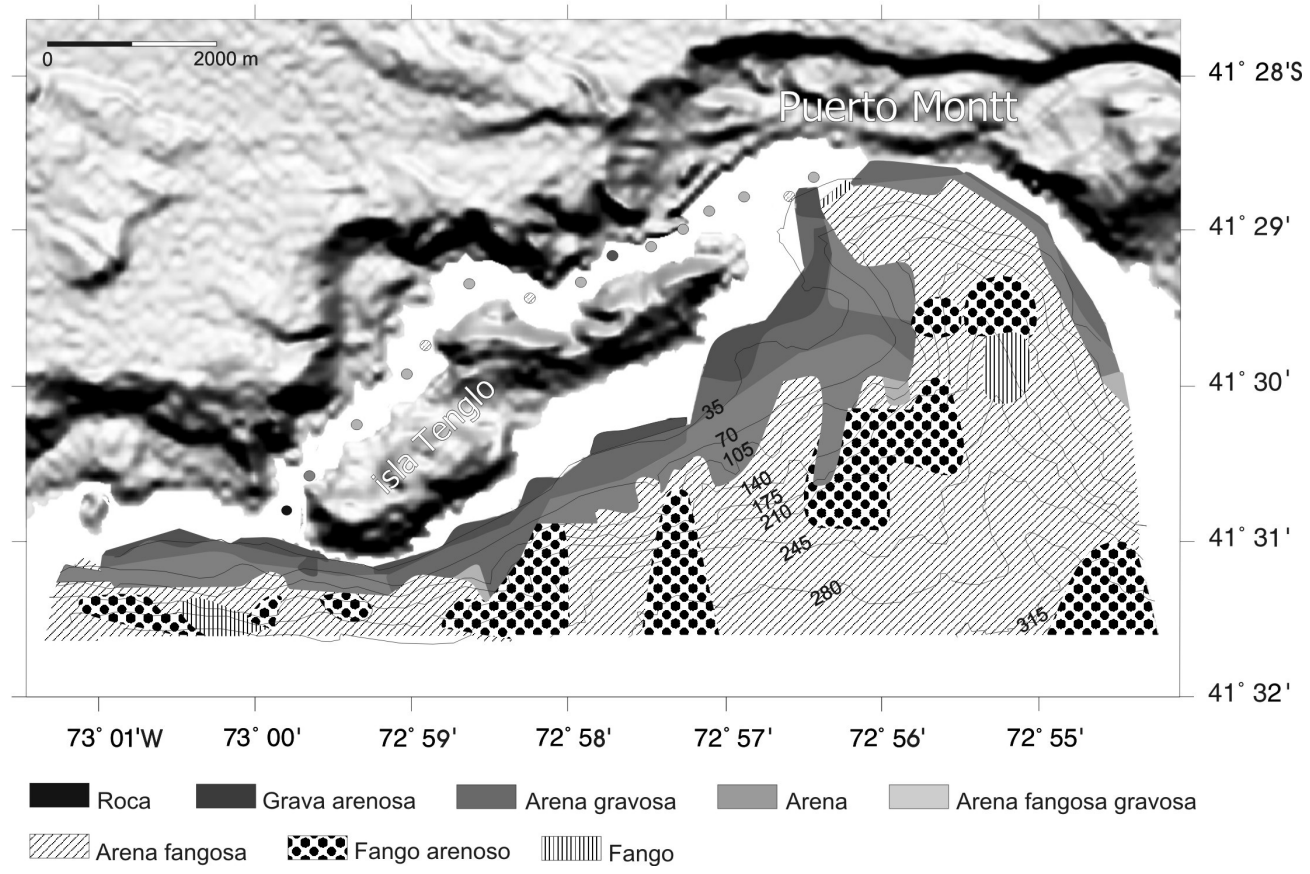

Figura 10. Mapa de la distribución de las características texturales de sedimentos superficiales a partir de la información de backscatter.

Figure 10. Textural sediment characteristic distribution map from backscatter information. 
están relativamente equidistantes de las bocas respectivas, indicando un solo mecanismo principal de depositación en estas áreas, que corresponde seguramente, a transporte por corrientes de marea. Sin embargo, otra área de fango se observó en el área central de la depresión o canal principal. Por lo tanto, parte del material fino es transportado desde la boca NE hasta ese sector, favorecido por la topografía y la pendiente del canal principal. Este canal principal se identifica como un área negra en la Figura 11. Como el canal principal nace en la boca NE del canal Tenglo, se sugiere una asociación geológica genética con la depresión que da origen a este canal. Además, varios canales submarinos menores desembocan en el área de fango sobre el canal principal (Fig. 11), lo que implicaría que el aporte sedimentario de material fino utiliza esos canales como conductos de sedimentos hacia sectores más profundos.

El mapa que interpreta el mosaico de sonogramas muestra las áreas con las variaciones principales de intensidad acústica de la señal del backscatter asociadas al fondo marino (Fig. 12). El área de fango mencionada en el párrafo anterior se presenta con una baja intensidad de las amplitudes de las señales acústicas. Lo mismo sucede en el área de fango del sector de la boca SW del canal Tenglo. Otras áreas cercanas con intensidades menores se localizan en las estructuras tipo terrazas de una forma convexa hacia mar adentro (Fig. 11). Este tipo de estructuras generalmente se asocian a una depositación por movimiento de masas (deslizamientos) provenientes de un sector más somero (Locat \& Lee, 2002).

En este sector de terrazas, el aporte de sedimentos provendría principalmente del canal Tenglo. Como no existen canales submarinos a continuación de estas terrazas, el material fino se va acumulando constantemente en esa zona. No ocurriría lo mismo en la boca NE que, dado que existe el canal principal, gran parte de lo depositado sigue su camino hacia el sur depositándose a profundidades mayores. Consecuentemente, los canales submarinos sirven de conductos de los materiales de aguas someras hacia el fondo.

Se encontró una asociación entre la distribución de materiales finos y la presencia de canales submarinos (Figs. 10 y 11). El sedimento de tamaño intermedio se transporta fácilmente por los canales (apegados al fondo) y se esparce en la zona profunda. Una parte del material fino debe quedar mezclado con material grueso y viajará a la zona profunda

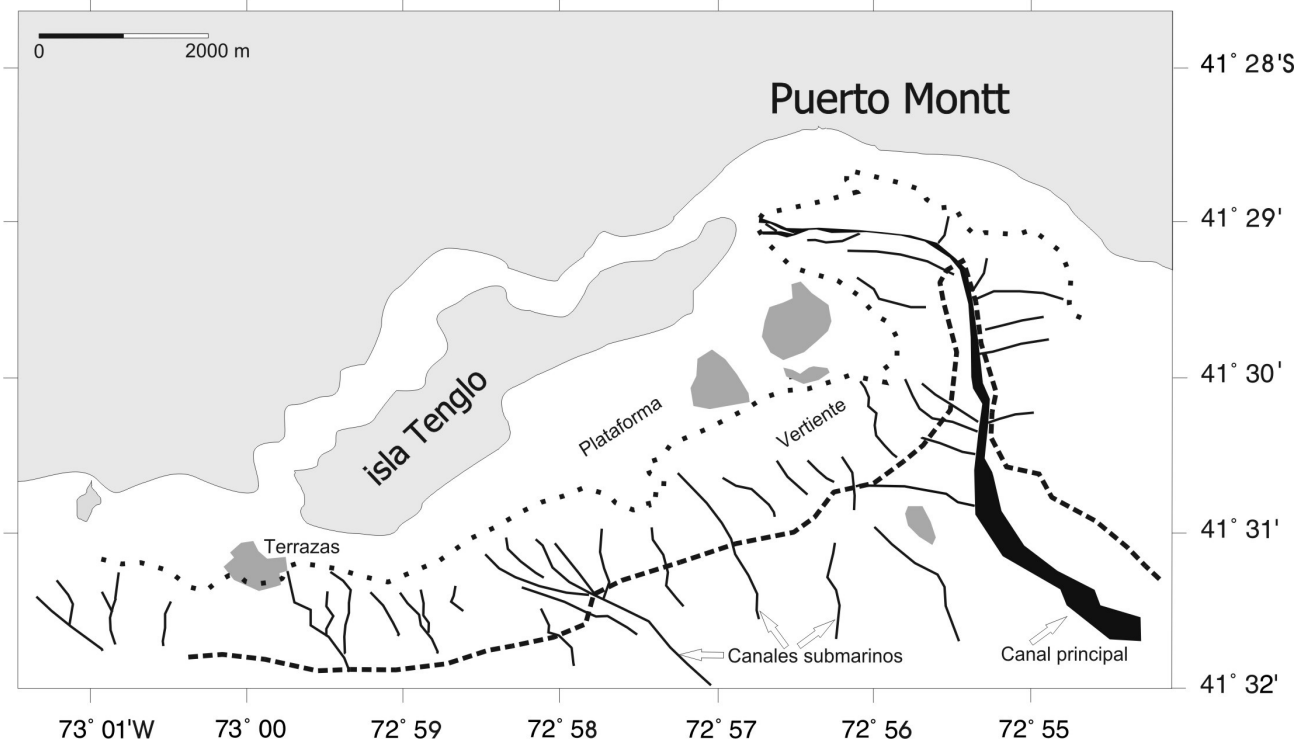

Figura 11. Interpretación del mapa de topografía submarina (Fig. 6). La línea de puntos indica el borde de la plataforma costera y la segmentada, el borde de la vertiente. Las líneas continuas indican canales submarinos, el área negra indica el canal o depresión principal. Las áreas grises indican zonas abultadas del fondo marino.

Figure 11. Submarine topography map interpretation (Fig. 6). Dot line shows coastal platform border and segmented line, slope border. Continued lines show submarine channels, black area shows main channel or depression. Grey areas show sea bottom bulky areas. 
como corrientes de turbidez a través de los canales submarinos. Otra parte debe quedar suspendida en la columna de agua y demorará un tiempo en sedimentar sobre el canal, lo que explicaría esta asociación en la distribución.

El material fino suspendido en la columna de agua, también será afectado por las corrientes y será llevado a otros sitios lejanos. Pero como se mostró anteriormente, se deposita cerca de los sectores de los canales. Entonces las corrientes no deben ser muy fuertes al interior de la bahía o los deslizamientos de materiales por la vertiente submarina y canales no son tan frecuentes. Hay que tener en consideración en la interpretación general, que puede existir una subestimación de la cantidad de sedimento fino, debido a la posible pérdida en el momento que la draga es izada desde el fondo.

Muchos autores encuentran una relación directa entre el tamaño de los granos y la fuerza de backscatter, donde los materiales más gruesos tendrían una mayor fuerza o intensidad del backscatter que los finos (Jackson et al., 1986b; Hughes-Clarke et al.; De Moustier, 2002). El backscatter responde al tamaño de grano, porque éste induce cantidades diferentes de rugosidad en el piso marino. En general, alta ru- gosidad se asocia a sedimentos gruesos (Borgeld $e t$ al., 1999; Urgeles et al., 2002).

En este trabajo la tendencia general encontrada confirma la relación anterior, además en el área de estudio existe una asociación entre la topografía submarina y las señales de backscatter (Figs. 6 y 12). El sector de la vertiente es más rugoso que la plataforma y la zona profunda, por lo tanto, tiene mayor intensidad o bien, no presenta gran cantidad de materiales finos. Luego, un parámetro importante a considerar en la intensidad del backscatter es la rugosidad del piso marino.

Sobre la plataforma existen áreas abultadas que tienen asociada una baja intensidad de backscatter (Figs. 11 y 12). Sin embargo, la Figura 10 indica que en esta área sólo existirían materiales gruesos. Desde el punto de vista morfológico, estas áreas son similares a aquellas encontradas en la boca SW del canal Tenglo (terrazas), lo que sugiere que pueden corresponder a un mismo tipo de material, es decir, sedimentos finos. Esto último estaría en concordancia con la baja intensidad del backscatter (modo side scan sonar, Figs. 8 y 12), que a pesar de ser abultadas, su parte superior puede adoptar una forma relativamente plana disminuyendo la intensidad acústica

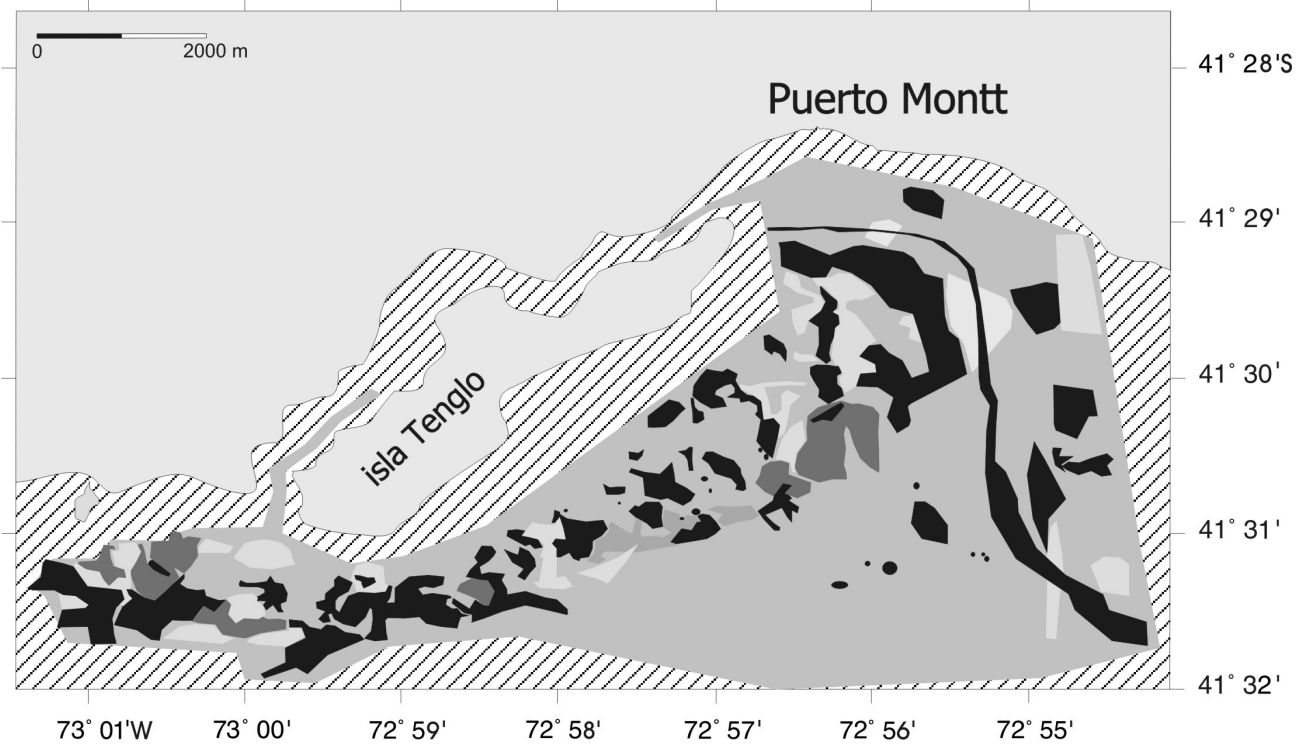

$<$ intensidad

$>$ intensidad

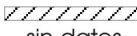
sin datos

Figura 12. Interpretación del mosaico de sonogramas. Las áreas en escala de grises se asocian a intensidades de las señales acústicas recibidas.

Figure 12. Mosaic sonogram interpretation. Areas with gray scales are associated to received signal intensity. 
recibida. Entonces, estas zonas corresponderían a acumulaciones de sedimentos más finos que las arenas que se ubican en los alrededores. La forma de estas áreas también sugiere que la depositación podría obedecer a un transporte favorecido por corrientes de marea que hace que parte del sedimento se acumule o mantenga en el lugar, no provocando un flujo o deslizamiento por la vertiente.

Sin embargo, en el sector de la plataforma pudieron haber ocurrido deslizamientos de porciones más grandes de materiales por la vertiente, esto es debido a la presencia de otras áreas abultadas entre la vertiente y la zona profunda de la bahía, las cuales tienen alta intensidad de backscatter (Figs. 11 y 12). Estos materiales pudieron provenir del borde de la plataforma (profundidad actual de 105 m, Fig. 6) y se deslizaron quizás por un sismo u otro mecanismo mecánico.

\section{AGRADECIMIENTOS}

Al Sr. Comandante C.F. Cristián Figari y tripulación 2002 del PSH "Cabrales", al Grupo Hidrográfico SHOA C1/2002, al Sr. Raúl Estrada por el análisis de las muestras de sedimentos, al Sr. Luis Flores y Sra. Wendy Rubio por su ayuda con las imágenes y a los Srs. Rodrigo Fernández y Stefan Kraus por sus observaciones. También quiero expresar mis agradecimientos a los tres revisores anónimos por sus valiosos comentarios.

\section{REFERENCIAS}

Atlas Electronics. 2001. Hydromap Atlas online. Bremen, $700 \mathrm{pp}$.

Augustin, J.M., R. Le Suave, X. Lurton, M. Voisset, S. Dugelay \& C. Satra. 1996. Contribution of the multibeam acoustic imagery to the exploration of the sea-bottom. Mar. Geophys. Res., 18: 459-486.

Borgeld, J.C., J.E. Hughes-Clarke, J.A. Goff, L.A. Mayer \& J.A. Curtiss. 1999. Acoustic backscatter of the 1995 flood deposit on the Eel River shelf. Mar. Geol., 154: 197-210.

Dartnell, P. \& J.V. Gardner. 2004. Predicting seafloor facies from multibeam bathymetry and backscatter data. Photogramm. Eng. Remote Sensing, 70: 1081-1091.

Recibido: 19 enero 2005; Aceptado 27 abril 2006
De Moustier, C. 2002. General echo-sounding theory in principles of multibeam echosounding (MBES). Notes of multibeam and high volume data processing workshop, Long Beach Campus, University of Southern Mississippi, 70 pp.

Folk, R.L., P.B. Andrews \& D.W. Lewis. 1970. Detrital sedimentary rock classification and nomenclature for use in New Zeland. N. Z. J. Geol. Geophys., 13: 937-968.

Gardner, J.V., M.E. Field, H. Lee \& B.E. Edwards. 1991. Ground-truthing $6.5 \mathrm{kHz}$ side scan sonographs: what are we really imaging. J. Geophys. Res., 96: 5955-5974.

Hughes-Clarke, J.E., B.W. Danforth \& P. Valentine. 1997. Aereal seabed classification using backscatter angular response at $95 \mathrm{kHz}$. In high frecuency acoustics in shallow water, NATO SACLANTCEN, Conference Proceedings Series CP-45: 243-250.

Jackson, D.R., D.P. Winebrenner \& A. Ishimaru. 1986. Application of the composite roughness model to high-frecuency bottom backscattering. J. Acoust. Soc. Amer., 79: 1410-1422.

Locat, J. \& H.J. Lee. 2002. Submarine landslides: advances and challenges. Can. Geotech. J., 39: 193-212.

Rabus, B., M. Eineder, A. Roth \& R. Bamler. 2003. The shuttle radar topography mission, a new class of digital elevation models acquired by spaceborne radar. Photogramm. Eng. Remote Sensing, 57: 241262.

Servicio Hidrográfico y Oceanográfico de la Armada (SHOA). 2001. Tabla de marea de la costa de Chile. SHOA, Valparaíso, Pub. 3009, 213 pp.

Urgeles, R., J. Locat, T. Schmitt \& J.E. HughesClarke. 2002. The july 1996 flood deposit in the Saguenay Fjord, Quebec, Canada: implications for sources of spatial and temporal backscatter variations. Mar. Geol., 184: 41-60.

Urick, R.J. 1983. Principles of underwater sound. McGraw-Hill, London, 448 pp.

Wentworth, C.K. 1922. A scale of grade and class terms for clastic sediments. J. Geol., 30: 377-392. 